\title{
Editorial: Advances in Time-Dependent Methods for Nuclear Structure and Dynamics
}

\author{
Lu Guo ${ }^{1}$, Denis Lacroix ${ }^{2}$, Nicolas Schunck ${ }^{3}$, Cédric Simenel ${ }^{4,5}$ and Paul Stevenson ${ }^{6 *}$ \\ ${ }^{1}$ School of Nuclear Science and Technology, University of Chinese Academy of Sciences, Beijing, China, ${ }^{2}$ Université Paris-Saclay, \\ CNRS/IN2P3, IJCLab, Orsay, France, ${ }^{3}$ Nuclear and Chemical Science Division, Lawrence Livermore National Laboratory, \\ Livermore, CA, United States, ${ }^{4}$ Department of Theoretical Physics, Research School of Physics and Engineering, Australian \\ National University, Canberra, ACT, Australia, ${ }^{5}$ Department of Nuclear Physics, Research School of Physics and Engineering, \\ Australian National University, Canberra, ACT, Australia, ${ }^{6}$ Department of Physics, University of Surrey, Guildford, United Kingdom
}

Keywords: nuclear physics, computational physics, time-dependent methods, nuclear reactions, nuclear structure

Editorial on the Research Topic

Advances in Time-Dependent Methods for Nuclear Structure and Dynamics

Among non-relativistic approaches to nuclear structure and reactions, the time-dependent Schrödinger equation is the basic equation from which microscopic approaches derive. Historically, stationary state methods which are not explicitly time-dependent have been preferred for describing both structure and reactions. One motivation for not directly solving time-dependent problems has been the computational difficulty, even if the physics case suggests retaining a time-dependent approach. Over time, such computational barriers have been reduced, and a general resurgence in time-dependent methods has occurred. In the last decade development of time-dependent mean-field codes has been substantial. Unrestricted 3D calculations with full effective interactions are now possible [1,2], and full treatment of superfluidity at the meanfield level has been included $[3,4]$. These worldwide efforts were a strong motivation to curate this Special Topic, which was conceived to draw together a snapshot of the current state of the wider field of time-dependent methods and their application in a broad range of problems in nuclear physics.

The article collection includes reviews as well as original material. Some directly address problems in the details of specific nuclei and in specific processes, while others deal with general problems, and general solutions, touching on fields outside nuclear physics.

In their contribution, Tokeida and Hagino address how one deals with open quantum systems. They start with a phenomenological description based on quantum friction and apply it to heavy-ion fusion. They then develop a more microscopic approach, based on a system-plus-bath Hamiltonian, appying it to the Caldeira-Legget model, with a discussion of how a future application to nuclear collisions could be made. Dinh et al. give a review of applications of time-dependent DFT methods to atoms, molecules and atomic clusters, concentrating on issues that are different from typical nuclear scenarios, but which could be relevant for future applications, such as direct laser-nucleus interaction [5].

Tohyama presents a review of the time-dependent density matrix method. This goes beyond the basic self-consistent microsopic approach of time-dependent Hartree-Fock (TDHF) by considering explicitly higher order terms in the BBGKY hierarchy, and Tohyama shows applications to both structure (excited vibrational states) and heavy-ion reactions. Also combining structure and reaction applications is Ebata's contribution, in which the details of the canonical-basis time-dependent Hartree-Fock Bogoliubov approach are reviewed and applied to resonances and heavy-ion reactions. Wang et al.'s review of semiclassical methods based on the time-evolution of the Wigner function 
show calculations of giant resonances, presenting results that combine dynamics with excited state structures, demonstrating the importance of nuclear collisions in determining excited state widths.

The topic of fission is covered in a review by Bulgac et al., giving an historical overview of methods used to tackle fission, details of the current state of the art, and a plan for the future. Fission is very much a current focus of time-dependent methods, and the review by Bulgac et al. complements a substantial review published recently elsewhere [6]. A method for describing spontaneous fission from a microscopic approach is given in a review by Sadhukhan. It brings together a WKB-like theory with Langevin dynamics in a framework suitable for making broad predictions of fission observables. An original research paper looking at details of fission is presented by Pancic et al., where the effect of the choice of underlying nuclear interaction is explored. A considerable part of the contribution from Verriere and Regnier concerns fission as described in the time-dependent generator coordinate method (TD-GCM), though they also highlight other processes and extensively review the theories behind different flavors of TD-GCM.

Studies of reaction mechanisms find a natural home in timedependent approaches, and several contributions are geared toward a better understanding of nuclear reactions. The minireview of Bao gives a critical analysis of different approaches to fusion reactions for production of superheavy nuclei; Godbey and Umar review some of the detailed observables that can be

\section{REFERENCES}

1. Maruhn JA, Reinhard P-G, Stevenson PD, Umar AS. The TDHF code sky3D. Comput Phys Commun (2014) 185:2195-216. doi:10.1016/j.cpc. 2014.04.008

2. Umar AS, Oberacker VE. Three-dimensional unrestricted timedependent hartree-fock fusion calculations using the full skyrme interaction. Phys Rev C (2006) 73:054607. doi:10.1103/PhysRevC.73. 054607

3. Jin S, Bulgac A, Roche K, Wlazłowski G. Coordinate-space solver for superfluid many-fermion systems with the shifted conjugate-orthogonal conjugategradient method. Phys Rev C (2017) 95:044302. doi:10.1103/PhysRevC.95. 044302

4. Scamps G, Hashimoto Y. Density-constrained time-dependent Hartree-FockBogoliubov method. Phys Rev C (2019) 100:024623. doi:10.1103/PhysRevC.100. 024623 understood in quasifission reactions using TDHF-based approaches; Jiang and Wang use a TDHF approach followed by a statistical model for deexcitation to look at reactions leading to new neutron rich isotopes.

Using time-dependent methods to link microscopic theories with collective models is a theme of Wen and Nakatsukasa who develop a new method to extract collective masses from microscopic calculations, and also of Washiyama and Sekizawa, who review the links between microscopic time-dependent methods and nucleus-nucleus potentials and friction coefficients in the Dissipation Dynamics-TDHF (DD-TDHF) method.

We mention finally the contribution of Iwata, which brings ideas from the theory of solitons to throw new light on the understanding of nuclear reactions making links to ideas perhaps less familiar in the nuclear theory community.

The response to this special topic has more than matched our expectations in terms of range and quality of contributions from the research community. We think it shows amply the strength and variety of developments and applications of time-dependent methods in nuclear physics, and beyond, and hope the resulting collection and e-book provide a useful reference for future developments.

\section{AUTHOR CONTRIBUTIONS}

All authors listed have made a substantial, direct, and intellectual contribution to the work and approved it for publication.

5. von der Wense L, Bilous PV, Seiferle B, Stellmer S, Weitenberg J, Thirolf PG, et al. The theory of direct laser excitation of nuclear transitions. Eur Phys J A (2020) 56:176. doi:10.1140/epja/s10050-020-00177-x

6. Bender M, Bernard R, Bertsch G, Chiba S, Dobaczewski J, Dubray N, et al. Future of nuclear fission theory. J Phys G Nucl Part Phys (2020) 47:113002. doi:10.1088/1361-6471/abab4f

Conflict of Interest: The authors declare that the research was conducted in the absence of any commercial or financial relationships that could be construed as a potential conflict of interest.

Copyright (C) 2021 Guo, Lacroix, Schunck, Simenel and Stevenson. This is an openaccess article distributed under the terms of the Creative Commons Attribution License (CC BY). The use, distribution or reproduction in other forums is permitted, provided the original author(s) and the copyright owner(s) are credited and that the original publication in this journal is cited, in accordance with accepted academic practice. No use, distribution or reproduction is permitted which does not comply with these terms. 